\title{
Unusual coloration in scarabaeid beetles
}

\author{
D J Brink ${ }^{1}$, N G van der Berg ${ }^{1}$, L C Prinsloo ${ }^{1}$ and I J Hodgkinson ${ }^{2}$ \\ ${ }^{1}$ Department of Physics, University of Pretoria, Pretoria 0002, South Africa \\ ${ }^{2}$ Department of Physics, University of Otago, Dunedin, New Zealand
}

Received 31 October 2006, in final form 31 January 2007

Published 16 March 2007

Online at stacks.iop.org/JPhysD/40/2189

\begin{abstract}
In this paper we investigate the reflection of circularly polarized light from the exocuticle of the scarabaeid beetle Gymnopleurus virens. Reflection spectra are deeply modulated, exhibiting a number of relatively narrow well-defined peaks, which differ from previously studied specimens. By comparing model calculations and electron microscopy work with the recorded spectra, we can propose the presence of specific structural defects responsible for the unusual spectra.
\end{abstract}

\section{Introduction}

The exceptionally bright and varied coloration in scarab beetles have long since fascinated people, such as the ancient Egyptians who regarded them as sacred objects. As long ago as 1911 Michelson [1] studied their unusual optical properties, such as their ability to reflect (mainly) left circularly polarized (LCP) light and not right circularly polarized (RCP) light.

A proper scientific basis for this effect was only provided in 1969 by Neville and Caveney [2] who showed that the outer part of the exocuticle of these insects consists of a regularly spaced layered structure where each layer is composed of a large number of parallel microfibrils. Each layer therefore acts as an optically anisotropic medium having a larger index of refraction for light polarized along the fibres (slow axis) than for perpendicularly polarized light (fast axis). In each subsequent layer the orientation of these axes is rotated by a small angle compared with the previous layer. This forms a helicoidal stack (usually left-handed) with a well-defined pitch (distance perpendicular to the layers for which a $360^{\circ}$ rotation is obtained). Circularly polarized light with the same pitch and handedness as the structure is then strongly reflected. However, unlike a normal mirror which changes the sense of rotation upon reflection, the reflection from the helicoidal structure retains the handedness of the circularly polarized light.

In recent years the optical properties of a number of such scarab beetles were studied [3-6]. In all the cases where reflection spectra were recorded these invariably showed relatively smooth broad structures. In contrast the Gymnopleurus virens beetle studied in this paper exhibits deeply modulated reflectance spectra with a number of relatively narrow well-defined peaks. In addition, this particular species is sometimes bright green and sometimes red (see figure 1). Both variations are common in many parts of Southern Africa with the green variation somewhat more

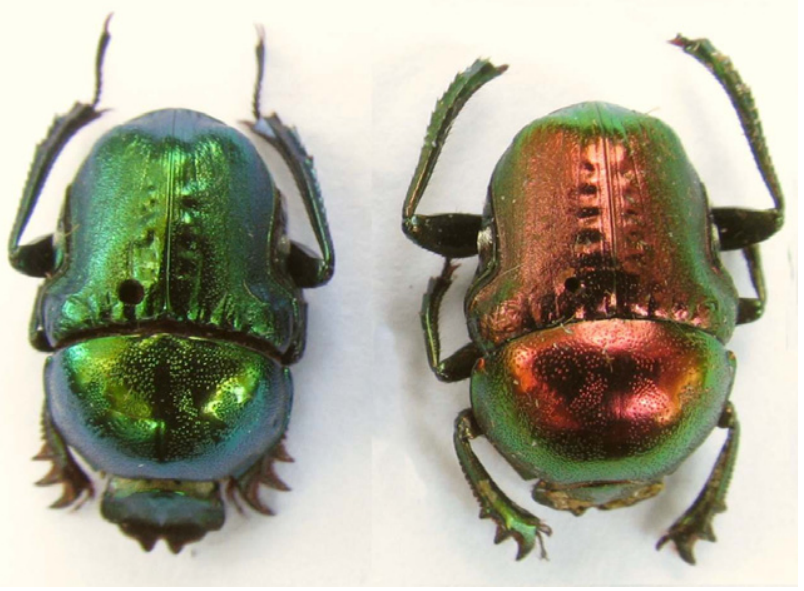

Figure 1. Photograph of the two Gymnopleurus virens beetles studied in this work. Note how the red changes to green at the edges of the red beetle when the angle of incidence increases. The same effect can be seen for the green beetle where green changes to blue around the edges.

abundant. It is interesting to note that the two types show characteristic differences in the modulation of their respective reflectance spectra.

Combining the angular dependence of the reflectance spectra, electron microscopy and model calculations, based on the Berreman $4 \times 4$ matrix formulation for anisotropic media (see section 2 ), we show that specific structural changes in the exocuticle can explain the observed spectra.

\section{Theoretical background}

Light propagation in an anisotropic medium can be described by casting the Maxwell equations into a $6 \times 6$ matrix form [7]: $R \Gamma=i k_{\mathrm{o}} M \Gamma$, where $\Gamma$ is a $6 \times 1$ column vector containing 
the spatial parts of the Cartesian components of the electric $(\boldsymbol{E})$ and the magnetic $(\boldsymbol{H})$ fields and $\boldsymbol{R}$ and $\boldsymbol{M}$ are $6 \times 6$ matrices containing the curl operator and the dielectric and magnetic permeability tensors, respectively. A time dependence of $\mathrm{e}^{+\mathrm{i} \omega t}$ is assumed and $k_{\mathrm{o}}$ is the vacuum propagation constant. Berreman [8] showed that this formulation can be simplified to a $4 \times 4$ matrix formulation when describing reflection and transmission of light from an anisotropic planar structure which is stratified along the $z$-axis. In this case the structure will appear locally uniaxial, with the slow axis $\left(n_{1}\right)$ along the fibrils in the $X Y$-plane at an angle $\beta z$ to the $x$-axis and two fast axes $\left(n_{2}=n_{3}\right)$, the first also in the $X Y$-plane at an angle $\beta z+90^{\circ}$ to the $X$-axis and the other along $Z$. All interfaces are assumed parallel to the $X Y$-plane and the plane of incidence of the light is chosen to be the $X Z$-plane. A plane-wave approximation is used where there is no variation along the $Y$ direction. For this special case:

$$
\frac{\partial \Psi}{\partial z}=-k_{\mathrm{o}} \Delta \Psi
$$

with $\Psi$ a generalized field vector containing only $x$ and $y$ components of $\boldsymbol{E}$ and $\boldsymbol{H}$ and $\Delta$ is the $4 \times 4$ Berreman propagation vector.

For a single domain cholesteric material, which is very similar to the helicoidal exocuticle structure studied here, Berreman showed that

$$
\Delta=\left[\begin{array}{cccc}
0 & 1-\frac{\eta^{2}}{\varepsilon_{\min }} & 0 & 0 \\
\varepsilon_{\mathrm{av}}+\delta \cos 2 \beta z & 0 & b \cdot \delta \sin 2 \beta z & 0 \\
0 & 0 & 0 & 1 \\
b \cdot \delta \sin 2 \beta z & 0 & \varepsilon_{\mathrm{av}}-\eta^{2}-\delta \cos 2 \beta z & 0
\end{array}\right]
$$

where $\varepsilon_{\min }$ and $\varepsilon_{\mathrm{av}}$ are the minimum and average values of the dielectric constant, $\delta$ is half the difference between the minimum and maximum dielectric constant values, $\eta=$ $n_{\mathrm{o}} \sin \phi_{\mathrm{o}}$ with $n_{\mathrm{o}}$ the ambient index of refraction and $\phi_{\mathrm{o}}$ is the angle of incidence and $\beta=2 \pi / P_{\mathrm{o}}$ with $P_{\mathrm{o}}$ the pitch of the helicoidal structure. This formulation is based on the approximation that the thickness of individual layers in the helicoidal structure approaches zero and it can handle both left- $(b=-1)$ and right- $(b=+1)$ handed structures.

In this work we obtained an expression for the spatial evolution of $\Psi$ by the direct integration of equation (1):

$$
\Psi(z+h)=\mathrm{e}^{-i k_{\mathrm{o}} h \Delta} \Psi(z)=L(h) \Psi(z) .
$$

The layer matrix $L$ was calculated by a suitable series expansion (up to nine terms) of the exponent [9].

Azzam and Bashara [7] showed that the layer matrix can be used to calculate a $2 \times 2$ reflection matrix for a slab of anisotropic material sandwiched between semi-infinite ambient and substrate layers for plane polarized incident light. It is straightforward to modify their calculation to obtain a reflection matrix for left-and right-circularly polarized light by a change of basis in the Jones vector formalism. This gives

$$
\boldsymbol{r}=\left[\begin{array}{ll}
r_{\mathrm{LL}} & r_{\mathrm{LR}} \\
r_{\mathrm{RL}} & r_{\mathrm{RR}}
\end{array}\right],
$$

where $r_{\mathrm{LL}}$ and $r_{\mathrm{RR}}$ are complex amplitude reflection coefficients for reflection of LCP light from incident LCP light and RCP light from incident RCP light, respectively. The other two terms represent cross reflections. Useful explicit expressions, based on the formulation of Azzam and Bashara [7], for the circularly polarized components in terms of the layer matrix components and the other system parameters are provided in the appendix. Different expressions for other formulations of the same problem can be found in the literature $[10,11]$.

Optimal reflection is obtained when the Bragg condition $[2,9]$ is met:

$$
\lambda=n_{\mathrm{av}} P_{\mathrm{o}} \cos \phi_{1}=2 n_{\mathrm{av}} P_{\mathrm{H}} \cos \phi_{1},
$$

where $\lambda$ is the vacuum wavelength at the reflection peak, $\phi_{1}$ is the angle of propagation inside the medium of average index of refraction $n_{\mathrm{av}}$ and $P_{\mathrm{o}}$ is the full pitch ( $360^{\circ}$ rotation) of the microfibrils. As the structure looks and behaves the same after a $180^{\circ}$ rotation, the Bragg condition is identical to that for a thin-film stack of layer thickness equal to the half-pitch value $P_{\mathrm{H}}$.

\section{Experimental details}

Samples were cut from the thorax of the insects as small regions (about $2 \times 2 \mathrm{~mm}^{2}$ ) could be found here which were locally quite flat and smooth.

Reflection spectra were recorded by illuminating sample material with a collimated beam (about $2 \mathrm{~mm}$ in diameter) of white light from a tungsten-halogen lamp. The incident and reflected beams could be left-or right-circularly polarized by passing it through plane polarizer and quarter-wave plate combinations. The input beam was focused on the sample to a spot of about $0.3 \mathrm{~mm}$ diameter and the reflected beam was passed through a $3 \mathrm{~mm}$ wide slit placed $60 \mathrm{~mm}$ from the sample. After this slit the light is focused on the entrance slit of the monochromator. This setup limits the angular spread of the reflected beam to less than $3^{\circ}$.

No attempt was made to measure absolute reflection values as biological samples invariably have non-perfect optical surfaces. Small deviations in flatness and a finite degree of surface roughness cause some light to be scattered outside the acceptance cone of the monochromator. The slit mentioned above fixes the reflection angles, but will introduce some loss in signal, which is hard to compensate for in a reliable way.

All recorded spectra were normalized by dividing point by point with the spectrum of the light source polarized in the same way as during recordings. This compensates for the wavelength-dependent detector response and monochromator transmission, but not for the scattering losses mentioned above. Spectra were recorded with a $0.64 \mathrm{~m}$ Jobin Yvon monochromator and a S20 photomultiplier.

Samples and illumination source were mounted on a turntable setup which allowed us to record spectra for a range of angles of incidence between $10^{\circ}$ and $65^{\circ}$. At larger angles the illumination was spread out too much which made it difficult to get the full spot on a sufficiently flat part of the sample.

Scanning electron microscopy work was done with a Joel 5800 microscope fitted with a Centaurus backscatter electron detector. Samples were embedded in Acryfix and then polished with diamond paste and rinsed in alcohol to expose a cross 


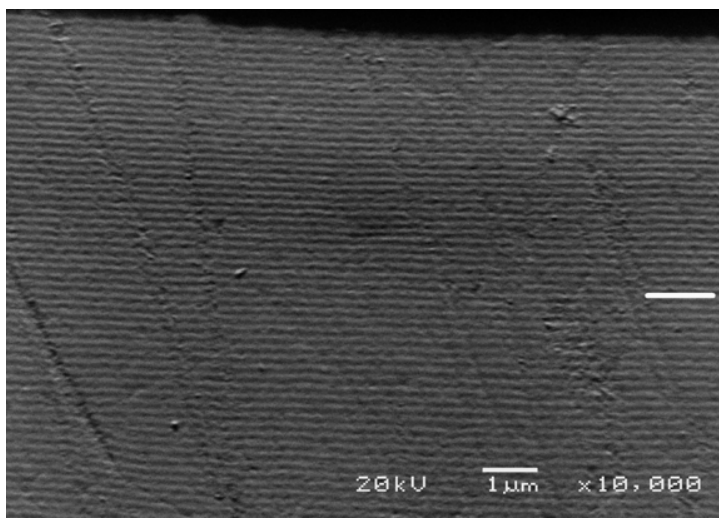

Figure 2. An electron micrograph of the cross section of the top part of the exocuticle of the green beetle. Note the increase in layer separation below the white marker at the right-hand edge.

section of the iridescent part of the exocuticle. Samples were then coated with a carbon layer followed by a gold-platinum layer to create conductive surfaces. The gold-platinum stream was incident on the sample at an angle of about $10^{\circ}$ to the surface in order to enhance the electron contrast due to the very slight undulations of the exposed surface [12].

We calibrated our quarter-wave plates against a Fresnel rhomb, where the fast axis corresponds to a 'P-type' reflection (electric vector in the plane of incidence), to ensure that the axes are correctly labelled and that left-and right-circularly polarized light are correctly identified. In this paper we use the usual convention that RCP light corresponds to a clockwise rotation when looking towards the source.

\section{Results and discussion}

\subsection{Electron microscopy}

A scanning electron microscope (SEM) picture of a cross section of the exocuticle of the green beetle is shown in figure 2. The spacing of the layers is roughly $0.17 \mu \mathrm{m}$. One can get an idea of how to interpret this by inserting the peak reflection wavelength (around $0.54 \mu \mathrm{m}$ ) into the Bragg condition (equation (5)) together with a reasonable guess for $n_{\text {av }}$ of about 1.6. This gives an approximate value for the pitch of $0.34 \mu \mathrm{m}$, which means that the SEM picture shows only two layers per pitch. Evidently every $180^{\circ}$ rotation of the microfibrils shows up as a single layer and the individual layers, comprising the actual helicoidal structure, are not resolved.

The pitch referred to here is the mechanical pitch $P_{\mathrm{o}}$, representing a rotation of $360^{\circ}$ in the orientation of the microfibrils. Obviously the structure looks the same physically and has the same dielectric properties after a $180^{\circ}$ rotation. It is therefore entirely reasonable that the period seen in the micrograph is the dielectric pitch $P_{0} / 2$.

Unfortunately no direct experimental evidence on the precise substructure of the helicoidal stack is available and the only evidence of its existence is a strong asymmetry in the left-right reflection coefficients. This occurs because the electric field associated with the standing optical wave in the chiral structure is locally locked to the fibril orientation in the finely layered substructure. A left-handed chiral structure

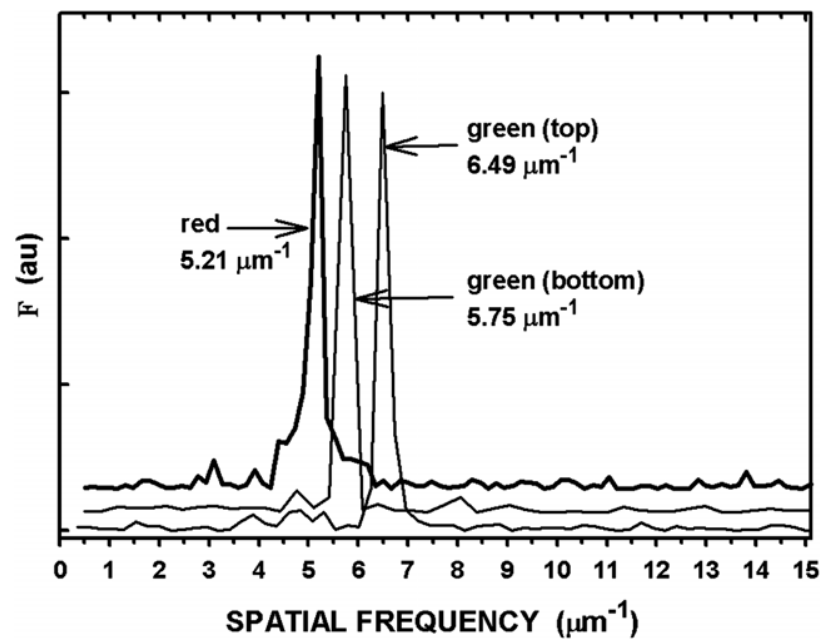

Figure 3. Fourier transforms of the electron micrographs for green and red beetles. The regions above and below the white marker on figure 2 exhibits well-defined distinct peaks, corresponding to half-pitch values of $0.155 \mathrm{mum}$ and $0.174 \mu \mathrm{m}$, respectively. For the red beetle the full active region produces a single peak corresponding to a half-pitch of $0.192 \mu \mathrm{m}$.

will therefore preferentially reflect a matching left-handed optical wave.

A close inspection of figure 2 shows that there is a sudden change in the layer spacing at the depth indicated by the white marker on the right edge. In order to quantify this accurately we performed a Fourier transform of the SEM pictures as shown in figure 3. As can be seen, the region above the marker exhibits a strong narrow peak at a spatial frequency of 6.49 lines $\mu \mathrm{m}^{-1}$, corresponding to a spacing of $0.155 \mu \mathrm{m}$. In the lower part of the picture the Fourier transform gives a well-defined peak, distinctly different from the top region. This peak is located at 5.75 lines $\mu \mathrm{m}^{-1}$ giving a spacing of $0.174 \mu \mathrm{m}$. This means that the pitch of the helicoidal system jumps by roughly $10 \%$. This discontinuity is seen in all electron micrographs of the green beetle. As will be pointed out in section 4.4, where model calculations are discussed, this period jump has an important influence on the width and structure of the reflectance band. Figure 3 also shows a spatial frequency spectrum for the red beetle taken over the full active region of the exocuticle. In this case, however, there is no clear evidence of more than one layer spacing.

\subsection{Angular resolution}

As a first experiment we established that the samples were sufficiently smooth and flat to regard reflections as specular. To this end we fixed the angle between the incident and reflected beams, carefully optimized the orientation of the sample for a maximum signal and then rotated the sample on its own, away from the optimum position. The result is shown in figure 4 . It is evident that the angular width of the reflection peak is mainly limited by the $3 \mathrm{~mm}$ slit between the sample and the monochromator (see section 3) and not by the sample surface itself. 


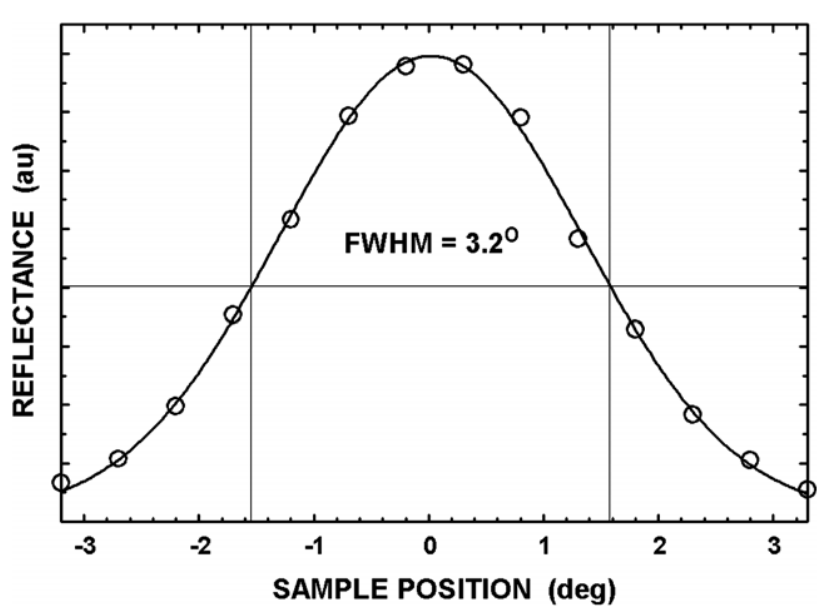

Figure 4. Change in reflectance as a sample of the red beetle is rotated away from the position of specular reflectance $\left(0^{\circ}\right.$ position $)$.

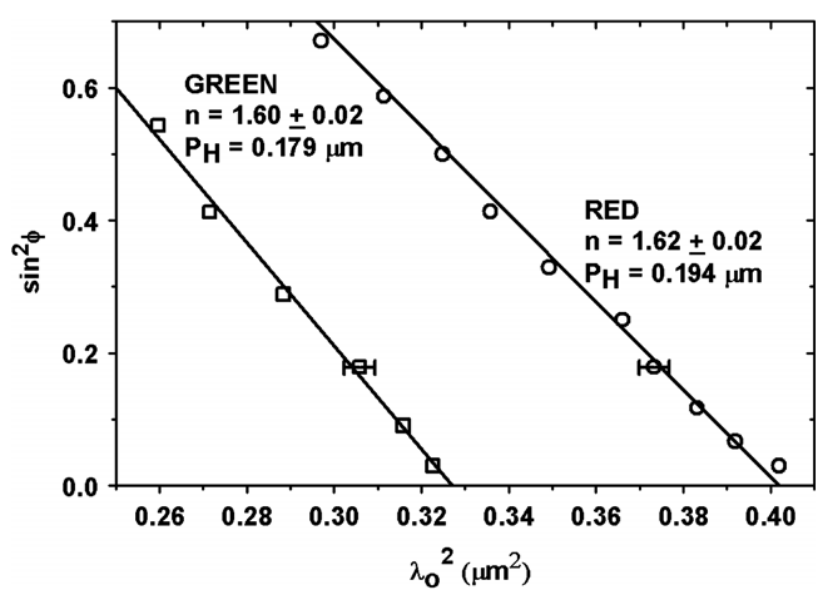

Figure 5. Straight-line fits for the $\sin ^{2}$ of the angle of incidence versus the square of the wavelength of a feature near the centre of the reflectance band. The shape of the reflection spectrum remained essentially unchanged during sample rotation. Parameter values are shown for linear regression lines.

\subsection{Optical determination of average index of refraction and pitch.}

According to the Bragg condition (see equation (5)) the reflectance spectrum will shift to the blue as the angle of incidence is increased. One can relate the internal angle $\phi_{1}$ to the external angle of incidence $\phi_{\mathrm{o}}$ by Snell's law: $n_{\mathrm{o}} \sin \phi_{\mathrm{o}}=$ $n_{\mathrm{av}} \sin \phi_{1}$, which changes equation (5) to

$$
\sin ^{2} \phi_{\mathrm{o}}=-\frac{1}{P_{\mathrm{o}}^{2}} \lambda^{2}+n_{\mathrm{av}}^{2}
$$

By fitting a straight-line to a $\sin ^{2} \phi_{0}$ versus $\lambda^{2}$ plot one can therefore obtain independent values for the pitch and the average refractive index of the structure.

Figure 5 shows the angular dependence of a spectral feature near the centre of the reflection band for the red and green beetles. As the difference between the two index of refraction values is of the same order of magnitude as the experimental error of \pm 0.02 , we decided to use a single average value of 1.61 for $n_{\mathrm{av}}$ for both beetles. In addition, as the
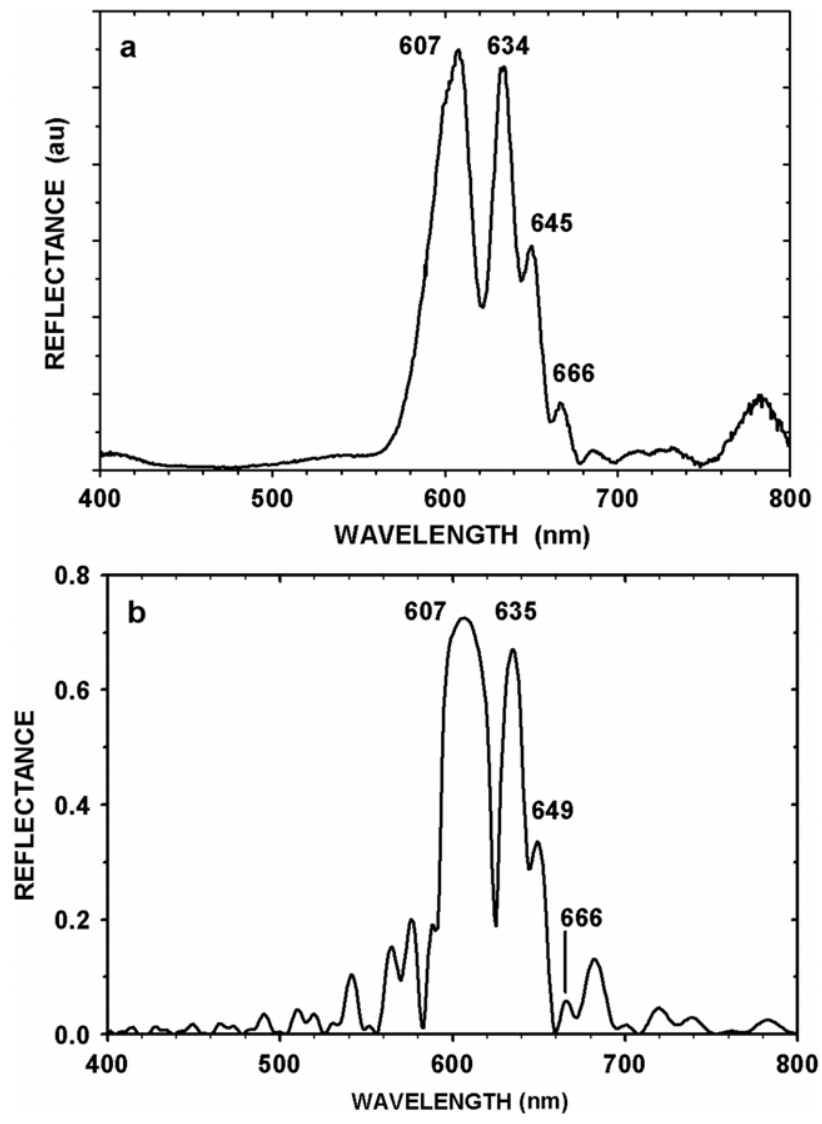

Figure 6. Experimental $(a)$ and modelled $(b)$ reflectance spectra for the red beetle at an external angle of incidence of $10^{\circ}$. Peak positions in $\mathrm{nm}$ are shown. Parameter values are $\delta n=0.065$, $P_{\mathrm{H}}=0.193 \mu \mathrm{m}$, structure thickness $=21$ pitches $=8.1 \mu \mathrm{m}$, depth of discontinuity $=5.0 \mu \mathrm{m}$, step in pitch $=0.6 \%$.

two specimens belong to the same species one would expect them to be constructed from the same material. The halfpitch value $\left(P_{\mathrm{H}}=P_{\mathrm{o}} / 2\right)$ of $0.194 \mu \mathrm{m}$, obtained for the red beetle, is probably representative and agrees well with the value of $0.192 \mu \mathrm{m}$ (see the $5.21 \mu \mathrm{m}^{-1}$ peak in figure 3 ) obtained from electron microscopy. For the green beetle the half-pitch value of $0.179 \mu \mathrm{m}$ should be interpreted as the average of the two sets of layers mentioned in section 4.1 and figure 2 $\left(P_{\mathrm{H}}\right.$ (average $\left.) \approx 0.164 \mu \mathrm{m}\right)$. This is about $9 \%$ larger than the electron microscopy values, but it should be more reliable as some biological samples are known to shrink slightly in vacuum (see Neville and Caveney [2]).

\subsection{Model calculations}

Typical experimental reflection spectra for the red and green beetles are shown in figures $6(a)$ and $7(a)$, respectively. Both spectra exhibit deep modulations resulting in a series of well-defined peaks. This is very different from the broad flat-topped reflectance spectra obtained with typical cholesteric materials [9] indicating the presence of some characteristic perturbation(s) in the helicoidal stacks of the beetle exocuticles. In addition the green reflectance band has a full width at half maximum (FWHM) of almost double that of the red band. Since the spectral width of a cholesteric material is given by $\delta \lambda=P_{\mathrm{o}} \delta n$ [9], with $\delta n$ the birefringence, this 

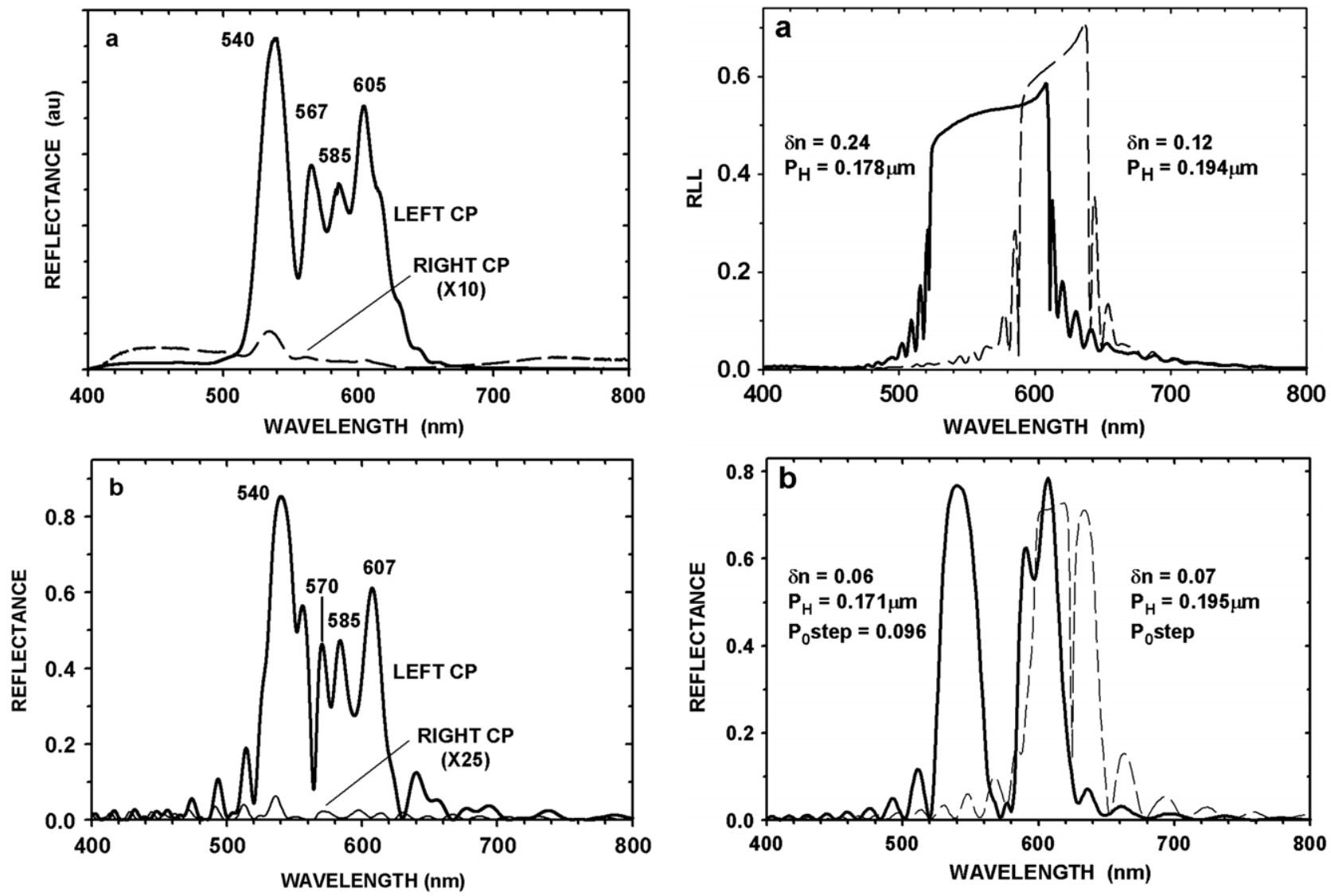

Figure 7. Experimental $(a)$ and modelled $(b)$ reflectance spectra for the green beetle at an external angle of incidence of $10^{\circ}$. Peak positions in nm are shown. Parameter values are $\delta n=0.065$, $P_{\mathrm{H}}=0.171 \mu \mathrm{m}$ (top), $1.85 \mu \mathrm{m}$ (bottom), structure thickness $=22$ pitches $=7.8 \mu \mathrm{m}$, depth of discontinuity $=4.2 \mu \mathrm{m}$, step in pitch $=8 \%$.

is rather strange as it indicates that the two colour variations are based on materials with a large difference in birefringence. One would expect beetles of the same species to be constructed from identical materials with at best a small difference in the layer thickness. The closely similar values for average refractive index also support this view.

A series of model calculations, based on the theory outlined in section 2, were performed in order to experiment with structure variations, which would bring calculated and measured reflectance spectra closer together.

Some results are shown in figure 8 . In a first calculation (figure 8(a)) unperturbed cholesteric media are assumed and the birefringence and half-pitch were adjusted until the FWHM and peak positions agreed with the experimental values. Halfpitch values of $0.178 \mu \mathrm{m}$ and $0.194 \mu \mathrm{m}$, agreeing well with the experimental values in section 4.3, were obtained for the green and red specimens, respectively. However, as pointed out above, the birefringence for the two cases differ by a factor of 2 and the value of $\delta n=0.24$ is unreasonably high for chitin. In this calculation the FWHM closely follows the expected $P_{\mathrm{o}} \delta n$ behaviour [9]. Apart from the problem with birefringence the spectra also lack the observed substructure and is clearly still far from a representative model.

In the second model, shown in figure $8(b)$, we included a step in the pitch of the structure at the position indicated

Figure 8. Modelled reflection spectra for a perfect cholesteric structure $(a)$ and a structure including a step in pitch $(b)$. The dashed lines represent red specimens and the solid lines green specimens.

by the white marker in figure 2. By using a somewhat larger step $(9.6 \%)$ for the green specimen than for the red $(1.3 \%)$ the correct spectral widths could be obtained with almost identical birefringence values (0.06 and 0.07). In addition both spectra now show strong modulations. For the red spectrum (dashed line) one can even get quite close to the experimental case by slightly tweaking the parameters $\left(\delta n=0.07, P_{\mathrm{o}}\right.$ step $\left.=1.3 \%\right)$. However, the green spectrum still contains too few peaks at the wrong spacing and with wrong relative heights. Also note that an increase in the pitch of $1 \%$, as predicted for the red specimen, is far too small to pick up on an electron micrograph, whereas the $9.6 \%$ jump for the green exocuticle is close to that seen in figure 2 .

As a final step, based on an idea from Hodgkinson and $\mathrm{Wu}$ [13], we included an abrupt $90^{\circ}$ rotation of the cholesteric structure at the same position as the period step. This is easily done by stepping $\beta$ in equation (2) by $90^{\circ}$ (or changing the sign of all sin- and cos-terms) at the same $z$-value where $P_{\mathrm{o}}$ is increased. This is perhaps not unreasonable as we are then assuming that the insect introduces both defects at the same point in its growth cycle.

As shown in figures $6(b)$ and $7(b)$, it is possible to obtain quite a close agreement between the modelled and the measured reflectance spectra by introducing this second perturbation to the structure. The few small remaining differences can possibly be ascribed to minor statistical variations in the structural parameters, which would tend to slightly smear out spectral features. 

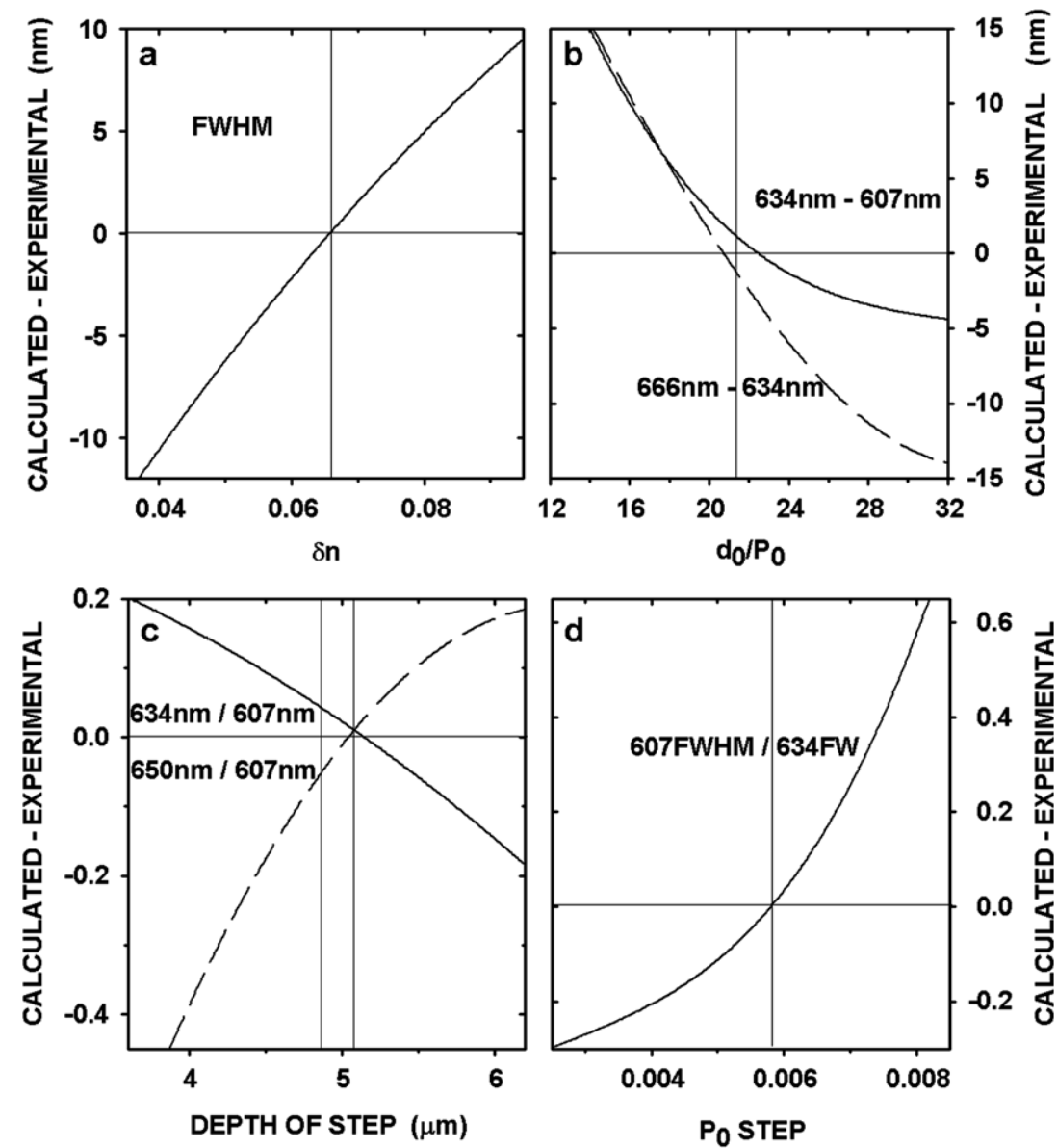

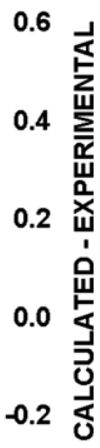

Figure 9. Influence of parameter values on the fit between experimental and modelled spectra for the red beetle. In $(a)$ the effect of birefringence $(\delta n)$ on the FWHM of the spectra is shown. The vertical axis gives the difference between the measured and modelled values. In $(b)$ the influence of the thickness of the active region $\left(d_{\mathrm{o}}\right)$ in terms of the pitch $\left(P_{0}\right)$ on the separation of the main peaks is shown. Once again the vertical axis shows the difference between the modelled and calculated values. The curves in $(c)$ show the effect of the depth at which the step in pitch occurs on the relative height of the main peaks. In $(d)$ the influence of the magnitude of the change in pitch, given as $\delta P / P_{\mathrm{o}}$, on the ratio of the widths of the main peaks is shown.

\subsection{Model parameters}

In order to get some quantitative idea of the accuracy of the proposed parameter values, we varied their values over a fair range around the best-fit values. This is shown in figures 9 (red beetle) and 10 (green beetle).

4.5.1. Red beetle. For the red specimen the only parameter significantly influenced by birefringence is the FWHM of the whole reflection band. Assuming an experimental error of about $\pm 1 \mathrm{~nm}$ in the peak positions a value of $\delta n=0.065 \pm$ 0.005 can be inferred from figure $9(a)$. In this case the $\delta n$ dependence of the FWHM is given by a slightly modified version of the simple relation mentioned in section 4.4 and [9]:

$$
\operatorname{FWHM}(\mu \mathrm{m})=0.025+P_{\mathrm{o}} \delta n,
$$

where the slope is still given by the product of the birefringence and the pitch, but a constant offset produced by the step in pitch should be added.

The total depth (length) of the active helicoidal structure determines the separation of the sub-peaks within the reflection band in a similar fashion to the role of the total thickness of a thin-film stack on its reflection spectrum. If the full thickness (about $15 \mu \mathrm{m}$ ) of the exocuticle is used a much too small spacing is obtained. As shown in figure $9(b)$ a best fit is obtained if the thickness of the cholesteric material is taken as 21 pitches, which is about $8.1 \mu \mathrm{m}$. We note that Neville and Caveney [2] also found that only roughly the top half of the exocuticle was optically birefringent and the lower part appeared to be more isotropic and perhaps weakly absorbing. This was built into our model by putting a 'soft' interface behind the cholesteric section. In the soft interface we allowed the birefringence ( $\delta n$ in equation (2)) to gradually decrease to zero over a distance of about $1 \mu \mathrm{m}$. The exact number of layers in the soft interface is of no importance. In this way there is no need to make any assumptions on the absorbing properties of the bottom isotropic part as it does not contribute to the reflection spectrum. From the results shown in figure $9(b)$ an accuracy of around one pitch length can be inferred.

As shown in figure 9(c) the depth where the defect occurs mainly influences the relative peak heights. A best fit is obtained for a depth between 4.8 and $5.1 \mu \mathrm{m}$. The magnitude of the step in the pitch length for the red beetle influences the asymmetry in the widths of the two main peaks. For an acceptable fit a step $\delta P_{\mathrm{o}} / P_{\mathrm{o}}=0.006 \pm 0.001$ should be used. 

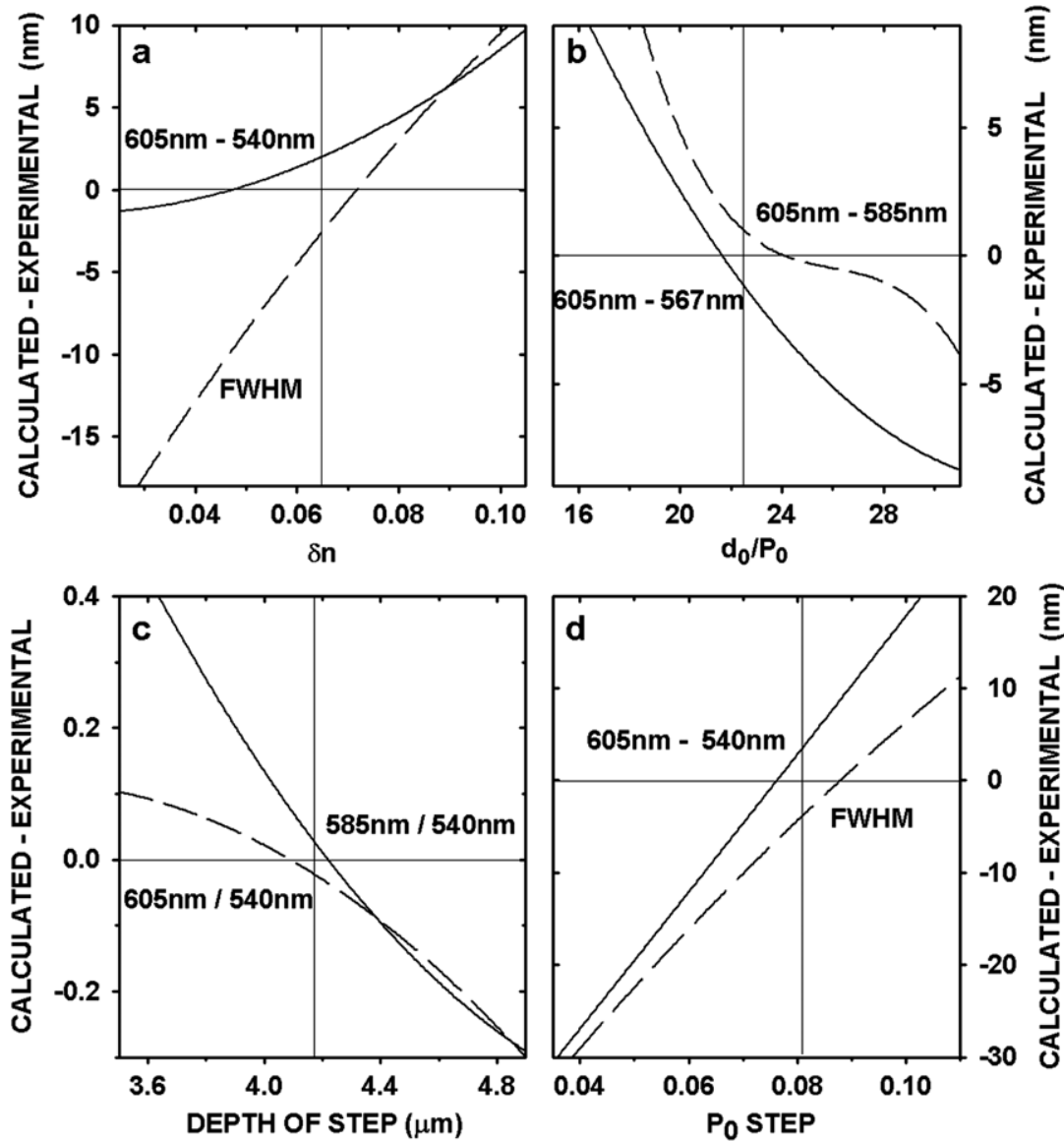

Figure 10. Influence of parameter values on the fit between experimental and modelled spectra for the green beetle. Curves shown in $(a)$, (b) and $(c)$ refer to the same quantities as in figure 9. In $(d)$ the influence of the magnitude of the change in pitch, given as $\delta P / P_{\mathrm{o}}$, on the FWHM and the separation of the main peaks is shown. As in figure 9 the vertical axis gives the difference between the experimental and calculated values.

4.5.2. Green beetle. For the green beetle parameters are to some extent coupled, and unambiguous best values are more difficult to obtain. As for the red specimen both the birefringence and the size of the step in the pitch length influence the FWHM, but the separation between the outer main peaks should also be considered. Assuming that the birefringence should be the same for both specimens $(\delta n=$ 0.065 ) as they are presumably made from the same material, the step in pitch is adjusted to give a best trade-off in these two features (see figure $10(d)$ ). Birefringence is now varied independently and, as shown in figure 10(a), a similar accuracy of \pm 0.005 is obtained. From figure $10(d)$ one obtains a relative change in the pitch of $\delta P_{\mathrm{o}} / P_{\mathrm{o}}=8 \% \pm 0.5 \%$, which is of the same order of magnitude as seen in the electron micrograph (figure 2). As can be expected, the FWHM is now somewhat more strongly affected by the step in pitch and the $\delta n$ dependence is now given by

$$
\operatorname{FWHM}(\mu \mathrm{m})=0.067+P_{\mathrm{o}} \delta n .
$$

The dependence on the magnitude of the $P_{\mathrm{o}}$-step is however not a simple linear function as we are dealing with interference between light reflected from two separate, slightly displaced sets of structures.
As shown in figure $10(b)$ the total thickness of the cholesteric part of the exocuticle is $22 \pm 1$ pitch lengths, which is very similar to that of the red specimen.

The influence of the depth position where the discontinuity occurs is shown in figure $10(c)$. It is a bit closer to the surface than for the red specimen at $4.2 \pm 0.1 \mu \mathrm{m}$.

Experimentally, a small amount of RCP light is also backscattered by the structure. This is partly due to the presence of a thin $(\approx 0.5 \mu \mathrm{m})$ transparent epicuticle on top of the exocuticle and presumably also due to small imperfections in the structure. As shown in figure 7(a) it amounts to less than $1 \%$ of the reflection for LCP light. As shown in figure $7(b)$ the model calculations, which also include a $0.5 \mu \mathrm{m}$ epicuticle (treated as a single uniaxial layer with the same $n_{1}$ and $n_{2}$ values as the helicoidal structure), are roughly in agreement with this. Similar results were observed for the red beetle.

\section{Conclusion}

We have shown that the Gymnopleurus virens beetle employs a form of defect engineering to broaden the reflectance band by between twice and four times the spectral width obtainable from perfect heliciodal stacks in the exocuticle. These defects also produce the deep modulations seen in the reflectance spectra. 
Different colours are achieved by members of the same species solely by thickness variations in the layered chiral structure.

Our observations of these scarab beetles may find practical applications in the fabrication of nano-engineered chiral reflectors used in display and laser technologies.

\section{Appendix}

Explicit values for the complex amplitude reflection coefficients for circularly polarized light. These formulae are valid for the same conditions as the formulation given by Azzam and Bashara [7] and Berreman [8], i.e. a monochromatic plane-wave approximation applied to an anisotropic medium sandwiched between two semi-infinite isotropic media. There is no restriction on the thickness to wavelength ratio.

$$
\text { If }
$$

$a_{1}=\cos \phi_{\mathrm{o}}\left(n_{2} L_{11}-\cos \phi_{2} L_{21}\right)$,

$a_{2}=i \cos \phi_{\mathrm{o}} n_{\mathrm{o}}\left(n_{2} L_{14}-\cos \phi_{2} L_{24}\right)$,

$a_{3}=n_{\mathrm{o}}\left(n_{2} L_{12}-\cos \phi_{2} L_{22}\right), \quad a_{4}=i\left(n_{2} L_{13}-\cos \phi_{2} L_{23}\right)$,

$A_{1}=a_{1}-a_{2}+a_{3}-a_{4}, \quad A_{2}=-a_{1}+a_{2}+a_{3}-a_{4}$,

$A_{3}=a_{1}+a_{2}+a_{3}+a_{4}, \quad A_{4}=-a_{1}-a_{2}+a_{3}+a_{4}$,

$b_{1}=\cos \phi_{\mathrm{o}}\left(n_{2} \cos \phi_{2} L_{31}-L_{41}\right)$,

$b_{2}=i n_{\mathrm{o}} \cos \phi_{o}\left(n_{2} \cos \phi_{2} L_{34}-L_{44}\right)$,

$b_{3}=n_{\mathrm{o}}\left(n_{2} \cos \phi_{2} L_{32}-L_{42}\right), \quad b_{4}=i\left(n_{2} \cos \phi_{2} L_{33}-L_{43}\right)$,

$B_{1}=b_{1}-b_{2}+b_{3}-b_{4}, \quad B_{2}=-b_{1}+b_{2}+b_{3}-b_{4}$,
$B_{3}=b_{1}+b_{2}+b_{3}+b_{4}, \quad B_{4}=-b_{1}-b_{2}+b_{3}+b_{4}$,

$G=A_{4} B_{2}-A_{2} B_{4}$,

then

$r_{\mathrm{LL}}=\frac{A_{1} B_{4}-A_{4} B_{1}}{G}, \quad r_{\mathrm{LR}}=\frac{A_{3} B_{4}-A_{4} B_{3}}{G}$,

$r_{\mathrm{RL}}=\frac{A_{2} B_{1}-A_{1} B_{2}}{G} \quad r_{\mathrm{RR}}=\frac{A_{2} B_{3}-A_{3} B_{2}}{G}$

where $n_{0}$ and $n_{2}$ are indices of refraction of the isotropic ambient and substrate layers and $\phi_{\mathrm{o}}$ and $\phi_{2}$ are the angles of incidence and refraction in the ambient and substrate layers, respectively. $L_{i j}$ represent elements of the layer matrix $\boldsymbol{L}$.

\section{References}

[1] Michelson A A 1911 Phil. Mag. 21 554-67

[2] Neville A C and Caveney S 1969 Biol. Rev. 44 531-62

[3] De Silva L, Hodgkinson I J, Murray P, Wu Q H, Arnold M, Leader J, and McNaughton A 2005 Electromagnetics 25 391-408

[4] Vigneron J P, Rassart M, Vandenbem C, Lousse V, Deparis O, Biro L P, Dedouaire D, Cornet A and Defrance P 2006 Phys. Rev. E 73 419051-8

[5] Hegedus R, Szel G and Horvath G 2006 Vis. Res. $462786-97$

[6] Goldstein D H 2006 Appl. Opt. 45 7944-50

[7] Azzam R M A and Bashara N M 1989 Ellipsometry and Polarized Light (Amsterdam: North Holland) pp 341-52

[8] Berreman D W 1972 J. Opt. Soc. Am. 62 502-10

[9] St John W D, Fritz W J, Lu Z J and Yang D K 1995 Phys. Rev. E 51 1191-8

[10] Ong H L 1987 Appl. Phys. Lett. 51 1398-400

[11] Ong H L 1988 Phys. Rev. A 37 3520-9

[12] Schwarz H and Gorb S 2003 Microsc. Res. Technol. 62 218-24

[13] Hodgkinson I J and Wu Q H 2001 Adv. Mater. 13 889-97 